4. Faraj AA, Omonbude OD, Godwin P. Gram staining in the diagnosis of acute septic arthritis. Acta Orthop Belg 2002;68: 388-91.

5. Carpenter CR, Schuur JD, Everett WW, Pines JM. Evidencebased diagnostics: adult septic arthritis. Acad Emerg Med Off J Soc Acad Emerg Med 2011;18:781-96. http://dx.doi.org/10. 1111/j.1553-2712.2011.01121.x.

Camillo Theo Müller ${ }^{a}$

Hand Surgery Unit, Department of Orthopeadic Surgery, Geneva University Hospitals and Faculty of Medicine, University of Geneva, Switzerland

E-mail addresses: camillotheo.mueller@hcuge.ch, camillo.mueller@gmail.com Ilker Uçkay ${ }^{a}$

Department of Orthopeadic Surgery, Geneva University Hospitals and Faculty of Medicine, University of Geneva, Switzerland

Department of Infectious Diseases, Geneva University Hospitals and Faculty of Medicine, University of Geneva,

Switzerland

Jean-Yves Beaulieu

Hand Surgery Unit, Department of Orthopeadic Surgery, Geneva University Hospitals and Faculty of Medicine, University of Geneva, Switzerland

(C) 2014 Published by Elsevier Ltd on behalf of British Association of Plastic, Reconstructive and Aesthetic Surgeons.

\section{Response to "The ulnar palmar perforator flap: Anatomical study and clinical application"}

\section{Dear Sir,}

We read with interest the article "The ulnar palmar perforator flap: anatomical study and clinical application" by $\mathrm{Hao}$ et al. ${ }^{1}$ The authors present an interesting novel approach to repair defects of the little finger and ulnar aspect of the hand, for which limited options are available.

We agree with the authors that perforator propeller flaps are an underexploited tool that provides a safe reconstruction with minimal donor site morbidity, and that their use should be encouraged. We thank the authors for providing anatomical and clinical evidence on the reliability of these flaps but we think that their paper deserves some comments for the sake of completeness.

\footnotetext{
${ }^{a}$ Both authors contributed equally to this publication. DOI of original article: http://dx.doi.org/10.1016/ j.bjps.2013.12.048
}

Perforator propeller flaps from the same source vessels - the ulnar palmar artery of the little finger- can also be harvested from the hypothenar region. Panse et al. reported an anatomical study and a clinical series for reconstruction of defects of the little finger, ${ }^{2}$ and we have further investigated their anatomy. ${ }^{3}$

Interestingly, constant perforators directed to volar and dorsal skin arise within a similar distance from the metacarpophalangeal joint. The option of harvesting the flap from the palmar surface can be advantageous for three reasons: bigger flaps can be raised (bigger flaps can be closed primarily and even healing by second intention is not an issue in the palm of the hand); scars on the dorsal and lateral ("social") surface of the hand are avoided; palmar skin will be reconstructed with palmar skin, respecting the like with like principle for the volar defects.

Once again, we'd like to thank the authors for their insight into the anatomy and the clinical potential of the perforator vessels from the ulnar palmar artery of the little finger; the potential of harvesting flaps from the palmar surface is an added benefit that allows for selection of the most appropriate perforator flap based on defect location and size.

\section{Conflict of interest}

None.

\section{Funding}

None.

\section{Ethical approval}

N/A.

\section{References}

1. Hao PD, Zhuang YH, Zheng HP, et al. The ulnar palmar perforator flap: anatomical study and clinical application. J Plast Reconstr Aesthet Surg 2014;67(5):600-6.

2. Panse N, Sahasrabudhe P. The ulnar digital artery perforator flap: a new flap for little finger reconstruction: a preliminary report. Indian J Plast Surg 2010;43:190-4.

3. Toia F, Marchese M, Boniforti B, Tos P, Delcroix L. The little finger ulnar palmar digital artery perforator flap: anatomical basis. Surg Radiol Anat 2013;35(8):737-40.

Francesca Toia

Plastic and Reconstructive Surgery,

Department of Surgical, Oncological and Oral Sciences, University of Palermo, Palermo, Italy

E-mail address: francescatoia@gmail.com Pierluigi Tos Department of Orthopedics and Traumatology, UOD Reconstructive Microsurgery, CTO-M. Adelaide Hospital of Turin, Turin, Italy 


\begin{abstract}
Adriana Cordova
Plastic and Reconstructive Surgery,

Department of Surgical, Oncological and Oral Sciences, University of Palermo, Palermo, Italy
\end{abstract}

(C) 2014 British Association of Plastic, Reconstructive and Aesthetic Surgeons. Published by Elsevier Ltd. All rights reserved.

http://dx.doi.org/10.1016/j.bjps.2014.05.035

\section{Rates of skin flap necrosis are increased in fat diabetic rats as compared to normal rat controls}

\section{Dear Sir,}

The rat is an often-used animal model in plastic surgery research. Multiple studies investigating flap physiology have utilized the well-described Modified McFarlane flap. ${ }^{1,2}$ This flap is created on the dorsum of the rat by incising three sides of a rectangle while leaving the pedicled base intact. The size often ranges between 2 and $3 \mathrm{~cm}$ in width and $7-10 \mathrm{~cm}$ in length. While creating the flap, the researcher elevates skin, subcutaneous tissue, and panniculus carinosus. Although there are many studies that have utilized these flaps in the basic rat species (Sprague-Dawley, Wistar), no studies exist on the manipulation of the species of rat itself. The Zucker Diabetic Fatty (ZDF) rat is an established animal model of both obesity and Type 2 Diabetes. ${ }^{3,4}$ The development of obesity and hyperglycemia-related complications is common between male ZDF rats and humans with type 2 diabetes associated with obesity. ${ }^{5}$ The authors of this investigation hypothesize that skin flap necrosis is higher in the ZDF rat as compared to the Wistar rat.

Rats were divided into two groups - the first included 17 Wistar rats and the second included $20 \mathrm{ZDF}$ rats. All rats were male, between the weights of 396-404 g, and were housed in a temperature-controlled environment. Both groups received normal saline subcutaneous injections once

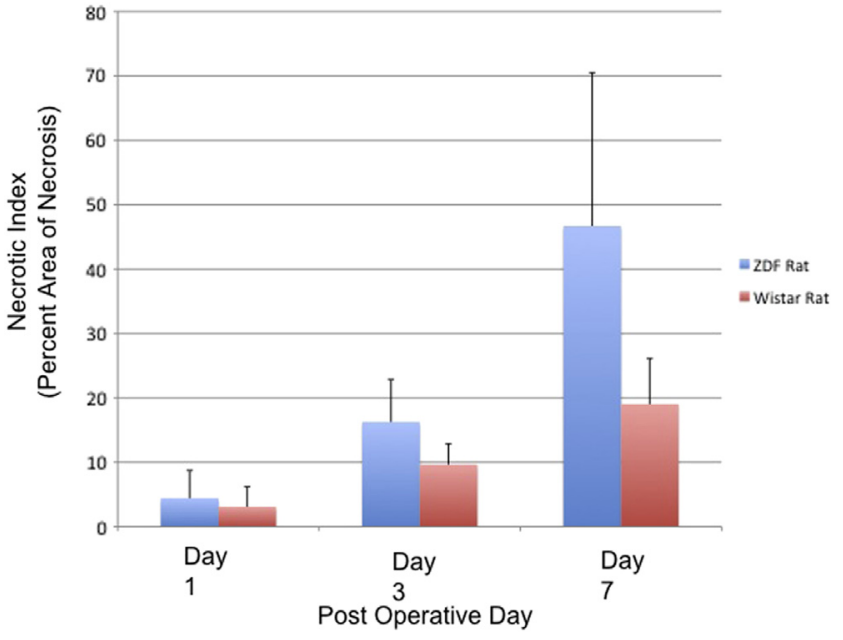

Figure 2 Graphical representation of results. There was nearly double the rate of necrosis seen in the ZDF rat group on day 3 , statistically significant $(9.63 \%$ vs $16.26 \%, p=0.0002)$. There was greater than double the rate of necrosis seen in the ZDF rat group on day 7 , statistically significant $(19.02 \%$ versus $46.70 \%, p=0.00002$ ).

daily for 28 days pre-operatively and 7 days postoperatively. After 28 days of pre-operative intervention, Modified McFarlane flaps were created on the dorsum of the rat $(3 \times 7 \mathrm{~cm})$. The base of the flap was situated between the iliac crests of the rat, and the flap was elevated below the panniculus carinosus. Silicone sheets were then interposed between the undersurface of the flap and the surrounding native tissue, and the flaps were sutured back into place. Rats were monitored for 7 days post-operatively, with digital photographs of each flap taken at postoperative day 1,3, and 7 (See Figure 1). Photographs were analyzed in a blinded fashion by 3 observers using Image J software. Necrotic index was calculated (area of necrosis/ total flap area). Statistics were performed using a $T$ Test analysis.

The calculated necrotic index of the Wistar rat group for days 1,3 , and 7 was $3.09 \%, 9.63 \%$, and $19.02 \%$ respectively. The necrotic index of the ZDF rat group for days 1,3 , and 7 was $4.42 \%, 16.26 \%$, and $46.70 \%$ (See Figure 2 ). There was

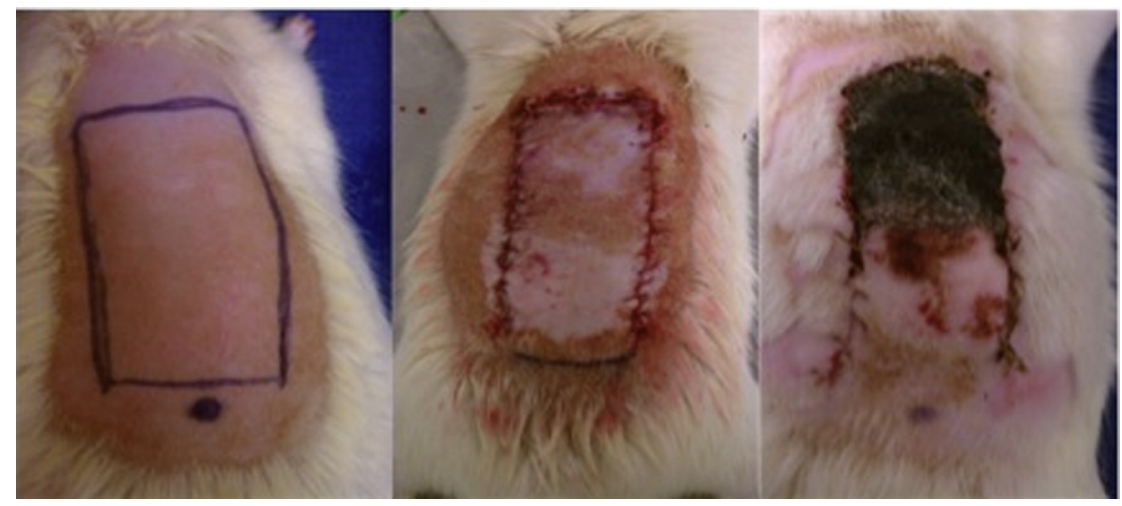

Figure 1 These photographs served as examples of the pre-operative planned McFarlane flap design, and the possible necrosis that could be seen post-operatively. 